\title{
BMJ Open Long-term clinical parameters after switching to nocturnal haemodialysis: a Dutch propensity-score-matched cohort study comparing patients on nocturnal haemodialysis with patients on three- times-a-week haemodialysis/ haemodiafiltration
}

To cite: Jansz TT, Özyilmaz A, Grooteman MPC, et al. Longterm clinical parameters after switching to nocturnal haemodialysis: a Dutch propensity-score-matched cohort study comparing patients on nocturnal haemodialysis with patients on three-timesa-week haemodialysis/ haemodiafiltration. BMJ Open 2018;8:e019900. doi:10.1136/ bmjopen-2017-019900

- Prepublication history and additional material for this paper are available online. To view these files, please visit the journal online (http://dx.doi org/10.1136/bmjopen-2017019900).

Received 2 0ctober 2017 Revised 19 December 2017 Accepted 3 January 2018
Check for updates

For numbered affiliations see end of article.

Correspondence to Dr Thijs Thomas Jansz; t.t.jansz@umcutrecht.nl

\section{ABSTRACT}

Objectives Nocturnal haemodialysis (NHD), characterised by 8 -hour sessions $\geq 3$ times a week, is known to improve clinical parameters in the short term compared with conventional-schedule haemodialysis (HD), generally $3 \times 3.5-4$ hours a week. We studied long-term effects of NHD and used patients on conventional $\mathrm{HD}$ / haemodiafiltration (HDF) as controls.

Design Four-year prospective follow-up of patients who switched to NHD; we compared patients with patients on HD/HDF using propensity score matching.

Setting 28 Dutch dialysis centres.

Participants We included 159 patients starting with NHD any time since 2004 , aged $56.7 \pm 12.9$ years, with median dialysis vintage $2.3(0.9-5.1)$ years. We propensity-score matched 100 patients on NHD to 100 on HD/HDF.

Primary and secondary outcome measures Control of hypertension (predialysis blood pressure, number of antihypertensives), phosphate (phosphate, number of phosphate binders), nutritional status and inflammation (albumin, $\mathrm{C}$ reactive protein and postdialysis weight) and anaemia (erythropoiesis-stimulating agent (ESA) resistance).

Results Switching to NHD was associated with a nonsignificant reduction of antihypertensives compared with HD/HDF (OR <2 types $2.17,95 \% \mathrm{Cl} 0.86$ to $5.50, \mathrm{P}=0.11$ ); and a prolonged lower need for phosphate binders $(\mathrm{OR}<2$ types $1.83,95 \% \mathrm{Cl} 1.10$ to $3.03, \mathrm{P}=0.02$ ). NHD was not associated with significant changes in blood pressure or phosphate. NHD was associated with significantly higher albumin over time compared with HD/HDF $(0.70 \mathrm{~g} / \mathrm{L} / \mathrm{year}$, $95 \% \mathrm{Cl} 0.10$ to $1.30, \mathrm{P}=0.02)$. ESA resistance decreased significantly in NHD compared with $\mathrm{HD} / \mathrm{HDF}$, resulting in a $33 \%$ lower ESA dose in the long term.

Conclusions After switching to NHD, the lower need for antihypertensives, phosphate binders and ESA persists for at least 4 years. These sustained improvements in NHD contrast significantly with the course of these parameters during continued treatment with conventional-schedule HD
Strengths and limitations of this study

- In this observational study on nocturnal haemodialysis, we used propensity-score matching as an expedient alternative to randomisation. Matched patients on nocturnal haemodialysis and controls were thus well comparable.

- Detailed data on several clinical parameters were collected for a long follow-up time in both cohorts prospectively.

- Primary outcomes included pill burden, which is an important factor in patient well-being.

- An important limitation is that this study was underpowered to evaluate mortality.

and HDF. NHD provides an optimal form of dialysis, also suitable for patients expected to have a long waiting time for transplantation or those convicted to indefinite dialysis.

\section{INTRODUCTION}

Nocturnal haemodialysis (NHD) has increasingly become a subject of research in recent years, as a potential solution for the high cardiovascular morbidity and mortality among haemodialysis (HD) patients. $^{1-3}$ Several factors are deemed responsible for this high risk. Recurrent states of hypervolaemia are known to cause left ventricular hypertrophy and cardiac remodelling. The consequent necessity of rapid fluid removal during dialysis may cause hypotension and compromise tissue perfusion. ${ }^{4-6}$ Furthermore, continuous hyperphosphataemia, often in conjunction with hypercalcaemia and hyperparathyroidism, leads to vascular calcifications. ${ }^{7-10}$ 
NHD provides opportunity for slower fluid removal and increased clearance of solutes such as urea and phosphate, ${ }^{11}$ due to twice as long (7-8 hours) dialysis sessions. ${ }^{12}$ Studies have shown beneficial effects of NHD on hypertension $^{13-18}$ and hyperphosphataemia, ${ }^{13} 1416-20$ yet data on nutritional status, anaemia control and mortality are not consistent. ${ }^{14}{ }^{16-21}$ In addition, as these parameters have not been investigated beyond 12 months of NHD treatment, it is not yet known whether these improvements last in the long run. We followed a large cohort of patients that switched to NHD prospectively, and compared longterm control of hypertension, phosphate and anaemia, as well as nutritional status to data collected in the same patients before switch to NHD. Then, we compared the long-term course of these parameters in NHD to a cohort of patients treated with conventional $(3 \times 3.5-4$ hours a week) HD and haemodiafiltration (HDF).

\section{METHODS}

\section{Outcomes}

We studied the following four domains as primary outcomes: hypertension control (predialysis systolic and diastolic blood pressure and number of different antihypertensive agents), phosphate control (phosphate and number of different phosphate binding agents), nutritional status and inflammation (albumin, $\mathrm{C}$ reactive protein (CRP) and postdialysis weight), and anaemia control (erythropoiesis-stimulating agent (ESA) resistance). We investigated all-cause mortality as secondary outcome.

\section{Cohorts}

We prospectively followed all patients who had switched to NHD at two major Dutch centres that offered NHD, as well as HD and HDF, either in-centre or at home with $\geq 7$-hour HD sessions. We defined baseline as the date of first NHD treatment, which was any time after April 2004. We collected data from electronic and paper records, from initiation until discontinuation of NHD, or until 1 February 2016. Ethical approval for this study was waived by the Medical Ethics Committee of the VU University Medical Centre, Amsterdam.

As reference, we used patient data from the CONvective TRAnsport STudy (CONTRAST, NCT00205556), a randomised trial designed to compare online HDF with low-flux HD regarding cardiovascular morbidity and mortality. CONTRAST randomised adult patients, treated with low-flux HD 2-3 a time weekly for at least 2 months with a single-pool Kt $/ \mathrm{V}_{\text {urea }} \geq 1.2$ per treatment, in a 1:1 ratio to treatment with low-flux HD or online HDF and followed for $1-7$ years. ${ }^{22}$ We used data of patients treated in the Netherlands (26 centres) with at least three sessions per week between June 2004 and January 2011.

\section{Data collection}

Apart from demographics and medical history, we collected data on clinical parameters and medication use at switch to NHD (baseline), and at 3, 6, 12, 18, 24, 36 and 48 months. Also, we recorded reasons for discontinuation of NHD. We recorded deaths that occurred within 3 months after cessation of NHD.

In CONTRAST, quarterly measured data similar to the NHD cohort were available for up to 48 months. Although mortality follow-up of CONTRAST was obtained irrespective of censoring, we adjusted these data to a follow-up similar to the NHD cohort (within 3 months of censoring).

In both cohorts, mean values of the last week before a selected time point were taken for predialysis blood pressure and postdialysis weight. Laboratory parameters were measured with routine assays. We converted albumin, when measured with bromocresol purple assays, to bromocresol green with the following formula ${ }^{23}$ :

$$
\text { Albumin }_{\text {bromcresol green }}=\text { Albumin }_{\text {bromcresol purple }}+5.5 \mathrm{~g} / \mathrm{L}
$$

We converted averaged weekly ESA doses of 1 month to defined daily doses (DDD) with conversion factors provided by the WHO Drug Classification. ${ }^{24}$

\section{Dialysis regimens}

We defined NHD treatment as $3-5 \times 8$ hours weekly either in-centre or at home, with a lower blood flow (150$220 \mathrm{~mL} / \mathrm{min})$, lower dialysate flow $(300 \mathrm{~mL} / \mathrm{min})$ and a somewhat lower bicarbonate concentration compared with conventional HD, adjusted depending on laboratory results. Anticoagulation was performed with low-molecular weight heparin (dalteparin or nadroparin) for in-centre NHD and unfractionated heparin for home NHD.

We defined conventional HD and HDF treatment as $3 \times 3.5-4$ hours a week (incidentally 5 hours), with blood flow rates between 300 and $400 \mathrm{~mL} / \mathrm{min}$. All patients used double-needle cannulation. Online HDF was performed in postdilution mode with a mean convection volume of $20.7 \pm 6.0 \mathrm{~L} /$ hour. $^{25}$ Anticoagulation was performed with low-molecular weight heparin. ${ }^{25}$

\section{Statistical analysis}

We performed longitudinal analyses with generalised linear mixed models, ${ }^{26}$ with time as continuous variable and random intercepts and slopes when appropriate. CRP and ESA resistance were skewed and therefore natural-log-transformed. For practical reasons, we dichotomised number of antihypertensive and phosphate-binding agents into $0-1$ and $\geq 2$ types; we present ORs for having a lower number of drugs compared with baseline ( $<2$ types). We performed survival analysis with Cox proportional hazards regression. ${ }^{27}$ We tested for proportional hazards assumptions with Schoenfeld's residuals.

To compare the NHD cohort with the HD/HDF cohort, we used propensity-score matching. In order to estimate propensity $\operatorname{scores}^{28}$, we imputed data missing at baseline 25 times $^{28}$ with multivariate imputations by chained equations. ${ }^{29}$ All variables had $\leq 1 \%$ missing values at baseline, apart from albumin $(1.8 \%)$, dialysis prescription $(2.4 \%)$, diabetes $(2.5 \%)$, smoking $(2.9 \%)$, residual 
glomerular filtration rate $(5.3 \%)$, cholesterol $(10.8 \%)$ and CRP $(30.7 \%)$. In each of the 25 imputed datasets, we matched patients on propensity of NHD treatment, ${ }^{30}$ logistically regressed on 22 variables affecting outcome or treatment selection (see online supplementary material). We matched nearest neighbours in a 1:1 ratio, without replacement, within a 0.1 calliper. We considered covariates balanced when standardised mean differences were $<0.1$.

We used multiple imputations only for propensity score matching. We performed longitudinal analyses for each matched cohort in the non-imputed dataset. We restricted analyses to the follow-up duration of each outcome available in the HD/HDF cohort. We pooled effect measures from the 25 analysed matched cohorts using Rubin's rules. ${ }^{28} 31 \mathrm{P}$ values $<0.05$ (two-sided) were considered statistically significant. All analyses were performed using R V.3.3.3. ${ }^{32}$

\section{Sensitivity analysis}

As sensitivity analysis, we repeated the multivariate imputations, propensity-score matching and subsequent analyses for NHD patients versus HD patients and NHD patients versus HDF patients separately, to account for potential benefits of HDF. As a post hoc sensitivity analysis, we adjusted for cause of end-stage renal disease (ESRD; being end-organ damage due to cardiovascular disease and diabetes mellitus), as this remained unbalanced after matching.

\section{RESULTS}

\section{NHD cohort at baseline $(n=159)$}

One hundred and fifty-nine patients from two large Dutch NHD centres $(n=76$ and $n=83)$ were included, representing an urban and a rural population. None of the patients had been treated with HDF previously. At baseline, mean age was $52.0 \pm 14.6$ years, $32 \%$ of patients were female, and median dialysis vintage was 2.5 (IQR $0.9-5.5$ ) years (table 1 ).

Twenty-nine per cent of patients were treated with home NHD, $86 \%$ of which used single-needle cannulation; $34 \%$ of in-centre NHD patients used single-needle cannulation. Median standard Kt/V (calculated with Leypoldt's formula ${ }^{33}$ ) was 3.02 (IQR 2.69-3.91). Seventy-eight patients discontinued NHD treatment within 2 years, due to renal transplantation (45\%), medical reasons (23\%), sleeping difficulties $(14 \%)$, social reasons $(6 \%)$, switch to diurnal home HD (4\%) and death (8\%).

\section{Baseline comparison of NHD and HD/HDF cohorts}

There were notable differences between the complete NHD and HD/HDF cohorts at baseline (table 1). We matched 200 (IQR 198-202) NHD and HD/HDF patients on propensity of NHD treatment. In the matched NHD cohort, mean age was $56.7 \pm 12.9$ years, and median dialysis vintage was $2.3(0.9-5.1)$ years at baseline. Twenty one per cent of patients had diabetes mellitus, and $31 \%$ a history of cardiovascular disease. Similar to the complete NHD cohort, patients were treated $7.8 \pm 0.4$ hours 3.5 (IQR 3.0-4.0) times a week. After matching, the NHD and HD/HDF cohorts were largely similar (table 2): age, sex, dialysis vintage, diabetes, history of cardiovascular disease, transplant waiting-list status, BMI and residual diuresis were balanced across both cohorts. Of note, cause of ESRD remained unbalanced after matching. We could not match $37 \%$ of the NHD cohort, mainly due to young age compared with the HD/HDF cohort. Hereafter, we refer to the matched cohorts as the NHD and HD/HDF cohorts.

\section{Longitudinal comparison of matched NHD and HD/HDF cohorts}

In the NHD cohort, systolic and diastolic blood pressure did not change over time (table 3). As can be seen from figure 1, blood pressure fluctuated somewhat during the third and fourth years in the HD/HDF cohort, but tended to decrease overall. There was no significant difference in systolic or diastolic blood pressure change between the NHD and HD/HDF cohorts (systolic blood pressure change vs HD/HDF: 1.94 (95\% CI -1.17 to 5.06) $\mathrm{mm} \mathrm{Hg}$ /year, $\mathrm{P}=0.22$, and diastolic blood pressure change vs $\mathrm{HD} / \mathrm{HDF}$ : 0.31 (95\% CI -1.31 to 1.93$) \mathrm{mm} \mathrm{Hg} /$ year, $\mathrm{P}=0.71$ ). In the NHD cohort, the number of antihypertensive agents diminished substantially after switching to NHD; the odds of having less antihypertensive agents increased over time (figure 1). Although the number of antihypertensive agents did not change in the HD/HDF cohort, the change in number of antihypertensive agents in the NHD cohort was not significantly different from the HD/HDF cohort (OR of <2 types vs HD/HDF per year: 2.17 (95\% CI 0.86 to 5.50$), \mathrm{P}=0.11$ ).

Initially, phosphate levels decreased slightly in patients who switched to NHD and remained stable during follow-up (figure 2). In patients on HD/HDF, phosphate levels did not decrease significantly and fluctuated during follow-up. The course of phosphate in NHD was not significantly different from that in HD/HDF $(-0.04$ (95\% CI -0.12 to 0.03$) \mathrm{mmol} / \mathrm{L} /$ year, $\mathrm{P}=0.23$ ). However, the number of phosphate-binding agents diminished sharply in patients who switched to NHD compared with patients on $\mathrm{HD} / \mathrm{HDF}$ ( $\mathrm{OR}<2$ types vs $\mathrm{HD} / \mathrm{HDF}$ per year: 1.83 ( $95 \%$ CI 1.10 to 3.03 ), $\mathrm{P}=0.02$, figure 2 ). Moreover, the absolute number of phosphate-binding pills decreased in the matched NHD cohort (from 6 at baseline (IQR 4-9) to 3 (IQR 0-5)) in the first 3 months after switching and remained stable afterwards.

In patients who switched to NHD, albumin was stable. Compared with patients on HD/HDF, NHD was associated with significantly higher albumin levels over time (0.70 (95\% CI 0.10 to 1.30$) \mathrm{g} / \mathrm{L} /$ year, $\mathrm{P}=0.02$; figure 3 ). CRP and postdialysis weight did not change significantly after switching to NHD, which was not significantly different from patients on HD/HDF (table 3).

ESA resistance decreased in patients who switched to NHD (ratio per year 0.61 (95\% CI 0.47 to 0.81 ), $\mathrm{P}<0.001$ ), while ESA resistance increased in patients on $\mathrm{HD} / \mathrm{HDF}$, 
Open Access

Table 1 Baseline characteristics of the complete (unmatched) NHD and HD/HDF cohorts

\begin{tabular}{|c|c|c|c|}
\hline & NHD (n=159) & HD/HDF $(n=560)$ & Standardised mean difference \\
\hline \multicolumn{4}{|l|}{ Demographics } \\
\hline Age (years) & $52.0 \pm 14.6$ & $64.0 \pm 15.2$ & 0.79 \\
\hline Male (\%) & 68 & 62 & 0.12 \\
\hline $\mathrm{BMI}\left(\mathrm{kg} / \mathrm{m}^{2}\right)$ & $26.1 \pm 6.2$ & $25.2 \pm 4.4$ & 0.18 \\
\hline \multicolumn{4}{|l|}{ Medical history } \\
\hline Dialysis vintage (years) & $2.5(0.9-5.5)$ & $2.0(1.0-3.8)$ & 0.22 \\
\hline \multicolumn{4}{|l|}{ Cause of ESRD (\%) } \\
\hline Glomerulonephritis & 24 & 13 & 0.63 \\
\hline Interstitial nephritis & 14 & 10 & \\
\hline Cystic kidney disease & 15 & 8 & \\
\hline Congenital, other & 5 & 1 & \\
\hline Renovascular & 17 & 30 & \\
\hline Diabetes mellitus & 9 & 15 & \\
\hline Multisystem disease & 6 & 5 & \\
\hline Other & 4 & 11 & \\
\hline Unknown & 6 & 9 & \\
\hline Current smoker (\%) & 16 & 21 & 0.13 \\
\hline \multicolumn{4}{|l|}{ Comorbidities (\%) } \\
\hline Diabetes mellitus & 21 & 21 & 0.00 \\
\hline Cardiovascular disease & 28 & 41 & 0.42 \\
\hline Transplant waiting-list listed (\%) & 23 & 33 & 0.21 \\
\hline \multicolumn{4}{|l|}{ Phosphate control } \\
\hline Phosphate (mmol/L) & $1.73 \pm 0.53$ & $1.64 \pm 0.50$ & 0.16 \\
\hline Different phosphate binding agents & $1.46 \pm 0.65$ & $1.27 \pm 0.74$ & 0.27 \\
\hline Vitamin D usage (\%) & 85 & 67 & 0.43 \\
\hline \multicolumn{4}{|l|}{ Hypertension control } \\
\hline Systolic blood pressure (mm Hg) & $140.4 \pm 21.2$ & $148.1 \pm 22.2$ & 0.36 \\
\hline Diastolic blood pressure (mm Hg) & $79.9 \pm 12.9$ & $76.1 \pm 12.4$ & 0.30 \\
\hline Different antihypertensive agents & $0.99 \pm 0.93$ & $1.55 \pm 1.28$ & 0.50 \\
\hline \multicolumn{4}{|l|}{ Nutritional status/inflammation } \\
\hline Postdialysis weight (kg) & $77.9 \pm 19.3$ & $72.4 \pm 13.9$ & 0.33 \\
\hline Creatinine $(\mu \mathrm{mol} / \mathrm{L})$ & $892 \pm 275$ & $886 \pm 249$ & 0.02 \\
\hline Albumin $(g / L)$ & $40.8 \pm 2.9$ & $40.0 \pm 3.8$ & 0.22 \\
\hline $\mathrm{CRP}(\mathrm{mg} / \mathrm{L})$ & $5.0(2.2-12.7)$ & $4.1(1.4-10.8)$ & 0.05 \\
\hline Cholesterol (mmol/L) & $3.9 \pm 1.1$ & $3.6 \pm 1.0$ & 0.26 \\
\hline \multicolumn{4}{|l|}{ Anaemia control } \\
\hline $\mathrm{Hb}(\mathrm{mmol} / \mathrm{L})$ & $7.1 \pm 0.8$ & $7.4 \pm 0.8$ & 0.33 \\
\hline ESA dose (DDD) & $7.8(3.9-13.3)$ & $8.9(4.4-13.3)$ & 0.18 \\
\hline ESA resistance index (DDD/Hb/kg/week) & $0.01(0.01-0.03)$ & $0.02(0.01-0.03)$ & 0.21 \\
\hline Use of iron supplementation (\%) & 88 & 75 & 0.34 \\
\hline \multicolumn{4}{|c|}{ Dialysis treatment parameters and residual kidney function } \\
\hline Residual diuresis > $100 \mathrm{~mL} / 24$ hours $(\%)$ & 45 & 50 & 0.11 \\
\hline Residual GFR (mL/min)* & $0(0-2.1)$ & $0.2(0-2.4)$ & 0.05 \\
\hline Central venous catheter (\%) & 10 & 1 & 0.38 \\
\hline Weekly dialysis sessions & $3.2 \pm 0.8$ & $3.0 \pm 0.1$ & 0.33 \\
\hline Weekly dialysis hours & $12.4 \pm 2.6$ & $11.3 \pm 1.2$ & 0.54 \\
\hline
\end{tabular}

${ }^{*}$ Mean of urea/creatinine clearance, 0 when residual diuresis $<100 \mathrm{~mL} / 24$ hours.

We report data as mean $\pm S D$, median $(I Q R)$ or proportions where appropriate; Standardised mean differences $<0.1$ are considered balanced.

BMI, body mass index; CRP, C reactive protein; DDD, defined daily dose; ESA, erythropoiesis-stimulating agent; ESRD, end-stage renal disease; GFR, glomerular filtration rate; $\mathrm{Hb}$, haemoglobin; $\mathrm{HD}$, haemodialysis; HDF, haemodiafiltration; NHD, nocturnal HD. 
Table 2 Baseline characteristics of the nocturnal haemodialysis (NHD) and haemodialysis/haemodiafiltration (HD/HDF) cohorts after propensity-score matching

\begin{tabular}{|c|c|c|c|}
\hline & NHD $(n=100)^{*}$ & HD/HDF $(n=100)^{*}$ & Standardised mean difference \\
\hline \multicolumn{4}{|l|}{ Demographics } \\
\hline Age (years) & $56.7 \pm 12.9$ & $56.3 \pm 15.1$ & 0.03 \\
\hline $\mathrm{BMI}\left(\mathrm{kg} / \mathrm{m}^{2}\right)$ & $25.9 \pm 5.6$ & $25.9 \pm 5.1$ & 0.01 \\
\hline \multicolumn{4}{|l|}{ Medical history } \\
\hline Dialysis vintage (years) & $2.3(0.9-5.1)$ & $2.1(1.1-4.0)$ & $<0.01$ \\
\hline Glomerulonephritis & 24 & 16 & 0.54 \\
\hline Interstitial nephritis & 13 & 6 & \\
\hline Cystic kidney disease & 16 & 9 & \\
\hline Congenital, other & 4 & 1 & \\
\hline Renovascular & 19 & 27 & \\
\hline Unknown & 5 & 9 & \\
\hline Current smoker (\%) & 19 & 19 & 0.02 \\
\hline \multicolumn{4}{|l|}{ Comorbidities (\%) } \\
\hline Diabetes mellitus & 21 & 19 & 0.02 \\
\hline Cardiovascular disease & 30 & 29 & 0.02 \\
\hline Transplant waiting-list listed (\%) & 28 & 28 & $<0.001$ \\
\hline \multicolumn{4}{|l|}{ Phosphate control } \\
\hline Phosphate (mmol/L) & $1.72 \pm 0.52$ & $1.69 \pm 0.53$ & 0.05 \\
\hline Different phosphate binding agents & $1.40 \pm 0.74$ & $1.43 \pm 0.64$ & 0.05 \\
\hline Vitamin D usage (\%) & 84 & 67 & 0.40 \\
\hline Creatinine $(\mu \mathrm{mol} / \mathrm{L})$ & $863 \pm 260$ & $938 \pm 273$ & 0.28 \\
\hline Albumin (g/L) & $40.6 \pm 3.0$ & $40.6 \pm 3.8$ & 0.02 \\
\hline $\mathrm{CRP}(\mathrm{mg} / \mathrm{L})$ & $5.0(2.3-13.6)$ & $3.7(1.4-10.1)$ & 0.02 \\
\hline Cholesterol (mmol/L) & $3.9 \pm 1.0$ & $3.9 \pm 1.0$ & $<0.01$ \\
\hline \multicolumn{4}{|l|}{ Anaemia control } \\
\hline $\mathrm{Hb}(\mathrm{mmol} / \mathrm{L})$ & $7.1 \pm 0.8$ & $7.4 \pm 0.7$ & 0.35 \\
\hline ESA dose (DDD) & $8.0(4.4-13.3)$ & $6.7(4.0-13.3)$ & 0.04 \\
\hline ESA resistance index (DDD/Hb/kg/week) & $0.01(0.01-0.03)$ & $0.01(0.01-0.03)$ & 0.01 \\
\hline Use of iron supplementation (\%) & 87 & 86 & 0.01 \\
\hline \multicolumn{4}{|c|}{ Dialysis treatment parameters and residual kidney function } \\
\hline Residual diuresis > $100 \mathrm{~mL} / 24$ hours (\%) & 49 & 50 & 0.04 \\
\hline Residual GFR (mL/min) $\dagger$ & $0.0(0.0-3.7)$ & $0.0(0.0-2.7)$ & 0.08 \\
\hline Central venous catheter (\%) & 4 & 5 & 0.04 \\
\hline Weekly dialysis sessions & $3.0 \pm 0.7$ & $3.0 \pm 0.1$ & 0.02 \\
\hline Weekly dialysis hours & $11.7 \pm 2.2$ & $11.6 \pm 1.1$ & 0.05 \\
\hline
\end{tabular}

*M edian 200 (IQR 198 - 202) matched cases.

†Mean of urea/creatinine clearance, 0 when residual diuresis $<100 \mathrm{~mL} / 24$ hours. We report data as mean $\pm \mathrm{SD}$, median (IQR) or proportions where appropriate. Standardised mean differences $<0.1$ are considered balanced.

$\mathrm{BMI}$, body mass index; CRP, $\mathrm{C}$ reactive protein; DDD, defined daily dose; ESA, erythropoiesis-stimulating agent; ESRD, end-stage renal disease; GFR, glomerular filtration rate; $\mathrm{Hb}$, haemoglobin; $\mathrm{HD}$, haemodialysis; $\mathrm{HDF}$, haemodiafiltration; NHD, nocturnal HD. 
Table 3 Effect estimates per year in the propensity-score-matched NHD and HD/HDF cohorts, and difference between the propensity-score-matched NHD and HD/HDF cohorts (all outcomes 48 months, except for CRP 36 months and ESA resistance index 12 months)

\begin{tabular}{|c|c|c|c|c|c|c|}
\hline & NHD & & HD/HDF & & NHD vs HD/HDF & \\
\hline Hypertension control & $\Delta$ & $\mathrm{P}$ & $\Delta$ & $\mathrm{P}$ & $\Delta$ & $\mathrm{P}$ \\
\hline Systolic blood pressure ( $\Delta \mathrm{mm} \mathrm{Hg}$ ) & $0.62(-1.74$ to 2.99$)$ & 0.27 & $-1.32(-3.40$ to 0.76$)$ & 0.22 & $1.94(-1.17$ to 5.06$)$ & 0.22 \\
\hline $\begin{array}{l}\text { Different antihypertensive agents } \\
\text { (OR<2 types) }\end{array}$ & 2.25 (1.12 to 4.54$)$ & 0.02 & 1.04 (0.55 to 1.97$)$ & 0.91 & 2.17 (0.86 to 5.50$)$ & 0.11 \\
\hline Phosphate control & $\Delta$ & $\mathrm{P}$ & $\Delta$ & $\mathrm{P}$ & $\Delta$ & $P$ \\
\hline $\begin{array}{l}\text { Different phosphate-binding agents } \\
\text { (OR <2 types) }\end{array}$ & 1.79 (1.13 to 2.84$)$ & 0.01 & $0.98(0.73$ to 1.31$)$ & 0.90 & 1.83 (1.10 to 3.03$)$ & 0.02 \\
\hline Nutritional status/inflammation & $\Delta$ & $\mathrm{P}$ & $\Delta$ & $\mathrm{P}$ & $\Delta$ & $\mathrm{P}$ \\
\hline Albumin $(\Delta \mathrm{g} / \mathrm{L})$ & $0.29(-0.14$ to 0.72$)$ & 0.19 & $-0.41(-0.85$ to 0.03$)$ & 0.07 & $0.70(0.10$ to 1.30$)$ & 0.02 \\
\hline CRP (ratio*) & 0.98 (0.91 to 1.06$)$ & 0.64 & 1.01 (0.90 to 1.13$)$ & 0.89 & 0.97 (0.84 to 1.12$)$ & 0.72 \\
\hline
\end{tabular}

Effect estimates are presented with $95 \% \mathrm{Cl}$.

${ }^{*} \mathrm{CRP}$ and ESA resistance were modelled with a gamma-distributed log link function. Hence, the (exponentiated) coefficients should be interpreted multiplicatively, that is, as a ratio.

CRP, C reactive protein; ESA, erythropoiesis-stimulating agent; HD, haemodialysis; HDF, haemodiafiltration; NHD, nocturnal HD.

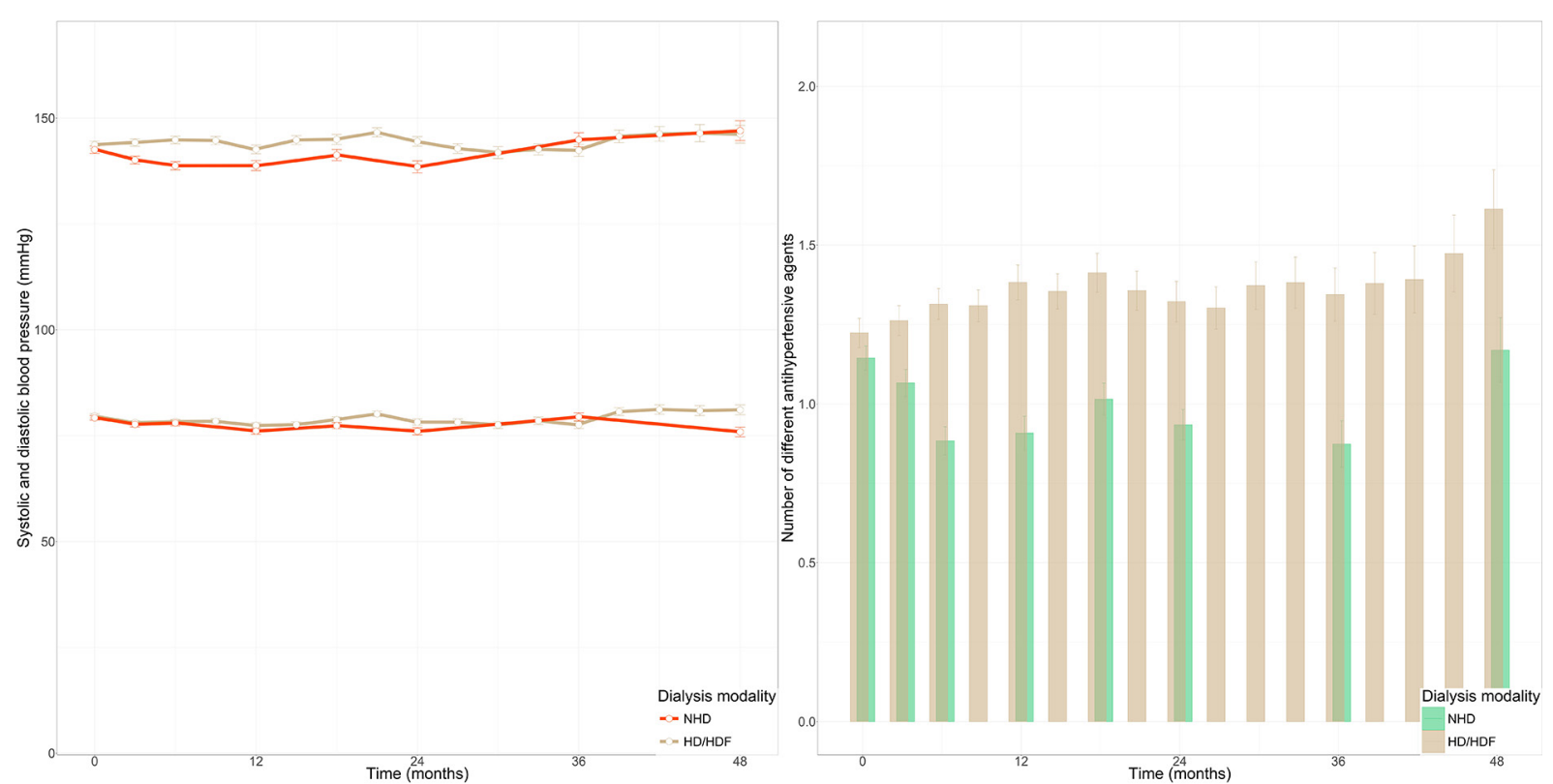

Figure 1 Hypertension control in nocturnal haemodialysis versus haemodialysis/haemodiafiltration. Left: systolic (upper two lines) and diastolic (lower two lines) blood pressure $(\mathrm{mm} \mathrm{Hg})$ in propensity-score-matched nocturnal haemodialysis (NHD, dark lines) and haemodialysis/haemodiafiltration (HD/HDF, light lines) patients over the course of 48 months. Right: number of different antihypertensive agents in propensity-score-matched NHD (dark line) and HD/HDF (light line) patients over the course of 48 months. OR $<2$ types NHD compared with baseline $\mathrm{P}=0.02$; $\mathrm{OR}<2$ types NHD vs HD/HDF $\mathrm{P}=0.11 .95 \% \mathrm{Cls}$ are shown. Number of NHD/HD/HDF patients available for analysis at 0 months: 100/100; 12 months: 57/74; 24 months: 35/51; 36 months: 20/34; 48 months: 11/22.

of whom only 1 year's data were available (figure 4 ). ESA resistance remained persistently low for up to 48 months after switching to NHD (ratio per year 0.89 (95\% CI 0.83 to $0.96), \mathrm{P}<0.01)$. ESA dose was reduced from median 8.0 (IQR 4.4-13.3) to 5.6 (IQR 2.2-8.9) DDD after 4 years of
NHD. Additional data on iron-storage parameters in both cohorts (ferritin, transferrin saturation), intravenous iron dose and residual urine production in the NHD cohort are available as online supplementary material. We provide data on the 4-year course of clinical parameters in the 


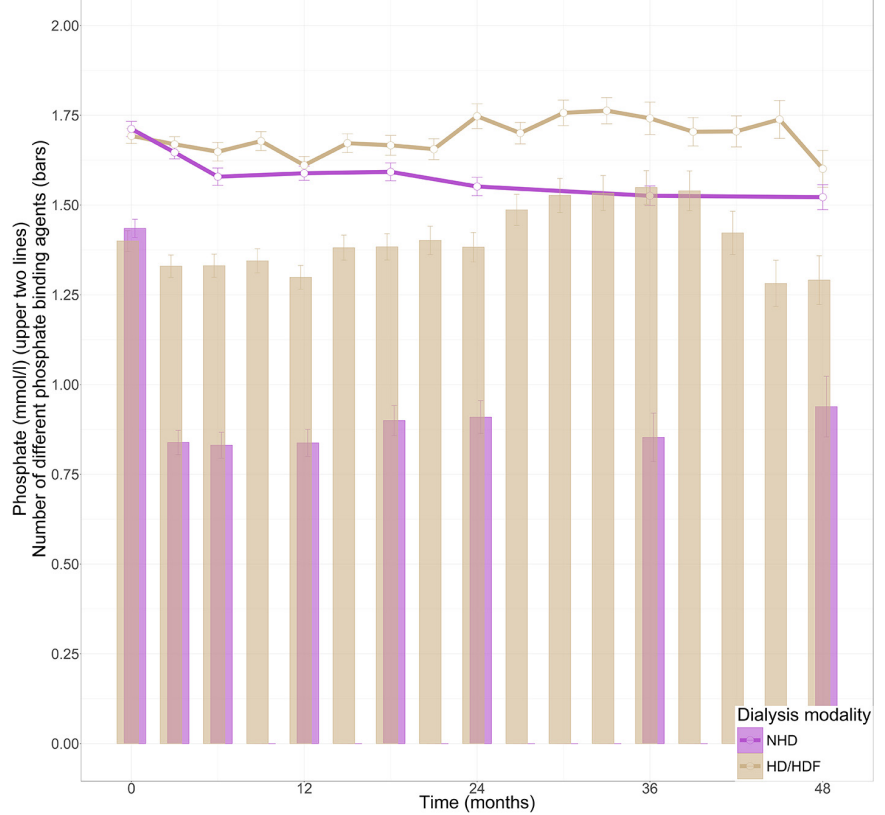

Figure 2 Phosphate control in nocturnal haemodialysis (NHD) versus haemodialysis/ haemodiafiltration (HD/HDF). Phosphate (lines, mmol/L) and number of different phosphate-binding agents (bars, same axis) in propensity-score-matched NHD (dark lines/bars) and HD/HDF (light lines/bars) patients over the course of 48 months. OR $<2$ types NHD compared with baseline $\mathrm{P}=0.01$; OR $<2$ types NHD vs HD/HDF $\mathrm{P}=0.02 .95 \% \mathrm{Cls}$ are shown. Number of NHD/HD/HDF patients available for analysis at 0 months: 100/100; 12 months: 57/74; 24 months: 35/51; 36 months: 20/34; 48 months: 11/22.

complete NHD cohort $(\mathrm{n}=159)$ as online supplementary material.

Thirty three NHD patients and $26 \mathrm{HD} / \mathrm{HDF}$ of the matched patients received a renal transplant, while 11 NHD patients and $23 \mathrm{HD} / \mathrm{HDF}$ patients died during a mean follow-up of $1.73 \pm 1.41$ and $2.28 \pm 1.38$ years. HR for all-cause mortality was $0.64(95 \% \mathrm{CI} 0.28$ to $1.48 ; \mathrm{P}=0.29)$ for NHD compared with HD/HDF patients.

\section{Sensitivity analysis}

When matching NHD with either HD (online supplementary table 1) or HDF patients (online supplementary table 2), similar results were obtained regarding hypertension, phosphate and anaemia control, nutritional status (online supplementary tables 3 and 4), and mortality (HR NHD vs HD 0.68 (95\% CI 0.29 to 1.59 ); HR NHD vs HDF 0.65 (95\% CI 0.28 to 1.49)). Also, post hoc sensitivity analysis with adjustments for causes of ESRD yielded similar results (online supplementary table 5).

\section{DISCUSSION}

To our knowledge, this is the first study to evaluate clinical parameters in detail for several years of NHD treatment. Our findings suggest that patients who switch to NHD experience long-term improvements of important

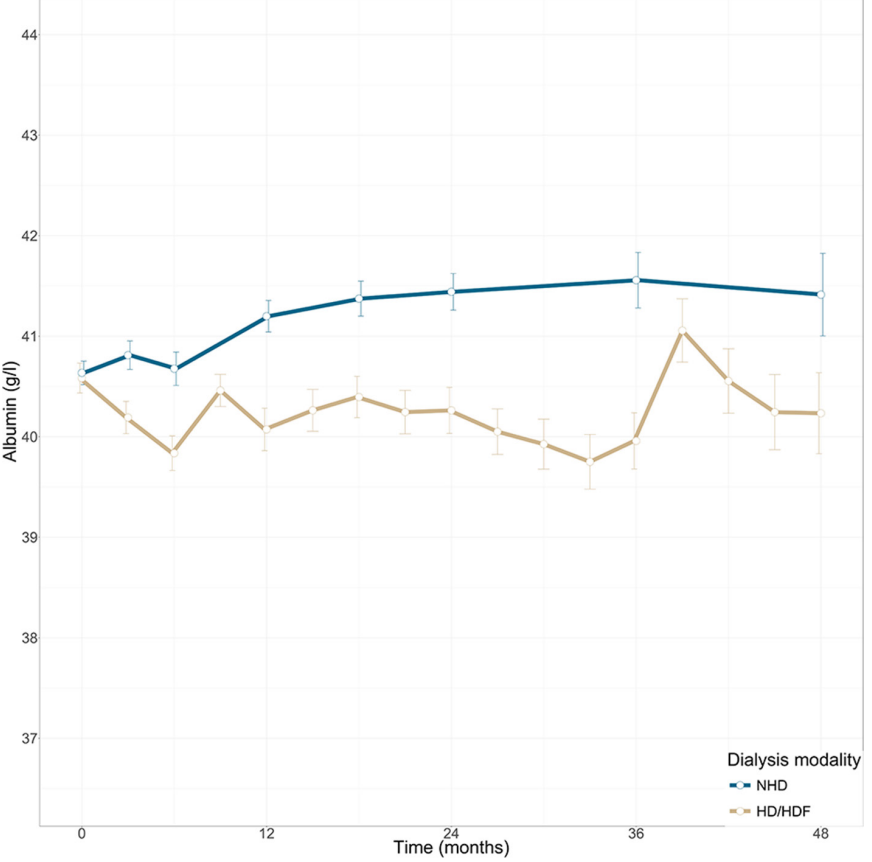

Figure 3 Albumin in nocturnal haemodialysis (NHD) versus haemodialysis/haemodiafiltration (HD/HDF). Albumin (g/L) in propensity-score-matched NHD (dark line) and HD/HDF (light line) patients over the course of 48 months. NHD compared with baseline $\mathrm{P}=0.19$; NHD vs HD/HDF $\mathrm{P}=0.02$. 95\% Cls are shown. Number of NHD/HD/HDF patients available for analysis at 0 months: 100/100; 12 months: 57/74; 24 months: 35/51; 36 months: 20/34; 48 months: 11/22.

clinical parameters, while benefiting from a greatly reduced pill burden and a lower need for ESA. As a randomised controlled trial for such a long follow-up is not feasible, with most patients refusing randomisation between such different dialysis modalities, we employed propensity score matching, the next-best method, for a well-founded comparison. Compared with patients who continued conventional treatment with $\mathrm{HD} / \mathrm{HDF}$, the above conclusions remained valid.

Our findings support more easily controlled hypertension in patients who switch to NHD, as they tend to develop a prolonged lower need for antihypertensive agents. Although the difference was not statistically significant compared with $\mathrm{HD} / \mathrm{HDF}$ patients $(\mathrm{P}=0.11)$, we consider the effect size clinically meaningful: NHD patients were over two times more likely to have their antihypertensives reduced from $\geq 2$ to 0 or 1 types. The lack of statistical significance may be due to the relatively small patient numbers and a loss of information by dichotomisation into $0-1$ and $\geq 2$ types of antihypertensives. We did not find explicit differences in blood pressure between NHD and HD/HDF patients, probably because systolic blood pressure did not decrease significantly in our NHD cohort, contrasting with some previous studies. ${ }^{131416}$ This may be explained by the heterogeneous NHD frequencies in our cohort: we observed significantly lower 1-year blood pressures in NHD patients who dialysed $>3$ times/ week compared with NHD patients who dialysed $\leq 3$ times/ 


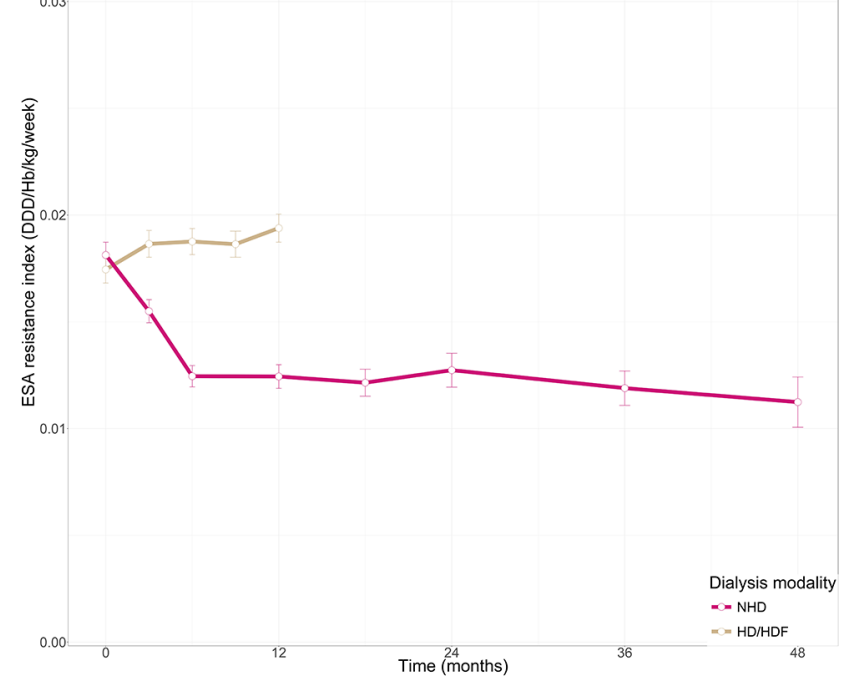

Figure 4 Erythropoiesis-stimulating agent (ESA) resistance in nocturnal haemodialysis (NHD) versus haemodialysis/ haemodiafiltration (HD/HDF). ESA resistance (DDD/Hb/kg/ week) in propensity-score-matched NHD (dark line) and HD/ HDF (light line) patients over the course of 48 months. NHD compared with baseline $\mathrm{P}<0.01$; NHD vs HD/HDF $\mathrm{P}<0.001$. $95 \% \mathrm{Cl}$ are shown. Number of NHD/HD/HDF patients available for analysis at 0 months: $100 / 100 ; 12$ months: 57/74; 24 months: 35/0; 36 months: 20/0; 48 months: 11/0. DDD, defined daily dose; Hb, haemoglobin.

week (data not shown). Consistent with this, a recent trial also did not find differences in blood pressure between extended-hours HD ( $\geq 24$ hours during mean $3.4 \pm 0.5$ sessions weekly) and conventional treatment. ${ }^{34}$

Evidently, NHD patients also had more easily regulated phosphate levels. Compared with HD/HDF patients, patients in the NHD cohort experienced a significantly lower need for phosphate-binding agents during the 48-month follow-up period. Also, the coinciding halving in absolute number of phosphate-binding pills in the NHD cohort corroborates the lower need for phosphate-binding agents in NHD patients, posing a great relief for their daily pill burden. As phosphate levels are often kept within target ranges ${ }^{35}$ by attending nephrologists, no outstanding differences in phosphate were observed. Thirty-four per cent of NHD patients had nearnormal phosphate levels $(\leq 1.50 \mathrm{mmol} / \mathrm{L})$ at baseline, compared with $52 \%$ at 12 months (with some receiving phosphate added to the dialysate).

As to nutritional status, we found striking differences between NHD and HD/HDF patients over the course of 48 months. In our study, NHD was associated with significantly higher albumin levels over time, compared with $\mathrm{HD} / \mathrm{HDF}$. This is in line with previous studies that described stable or even increasing albumin levels following initiation of NHD. ${ }^{14} 1619$ CRP levels did not differ significantly between the NHD and $\mathrm{HD} / \mathrm{HDF}$ patients. Postdialysis weight remained stable in NHD patients over the years. Contrary to what we had expected based on previous reports, ${ }^{16}{ }^{19}$ this was not significantly different from HD/HDF patients, which might be explained by patients adopting a more active lifestyle after several years on NHD. NHD patients also achieved superior anaemia control compared with $\mathrm{HD} / \mathrm{HDF}$ patients, contrasting with previous conflicting reports. ${ }^{13} 14161819$ Although haemoglobin did not change in NHD patients, presumably due to adjustments of ESA and iron dosages according to guidelines, ESA resistance diminished evidently in NHD patients and remained persistently low for up to 4 years, against a clear increase in ESA resistance in the HD/HDF cohort.

Amid conflicting data, a recently published large study showed a lower mortality risk in patients treated with extended-hours dialysis compared with conventional HD (HR 0.67 (95\% CI 0.49 to 0.93$)$ ). ${ }^{36}$ We found a remarkably similar lower mortality risk in NHD (HR 0.64 (95\% CI 0.28 to 1.48$)$ ), although our study was not powered to prove mortality differences due to its design and consequent sample size. It should be noted that no safety events (ie, vascular access complications) were recorded, as we did not expect additional complications because of the habit of single-needle cannulation in frequent NHD.

Remarkably, more NHD patients were transplanted compared with matched $\mathrm{HD} / \mathrm{HDF}$ patients (32.6 vs $26.3 \%$ ), despite similar rates of transplant waiting-list listing at baseline. As transplanted, presumably healthier patients dropped out more frequently in the NHD cohort. This could have resulted in a somewhat less healthy matched cohort, which could have led to an overestimation (but surely no underestimation) of mortality hazard in the NHD cohort. The reasons for the disparate transplantation rates remain unclear. Possibly, clinical improvement of non-waiting-list-listed NHD patients resulted in more transplant waiting-list-listed patients.

There were limitations to this study, and the results should be interpreted within the limitations of this study. First, this study was non-randomised, and as a result, patients in our NHD cohort were from a selected population, as with many other studies on NHD. ${ }^{17}{ }^{19}$ We used propensity-score matching to reduce this selection bias, so that the groups were similar in baseline characteristics. Although causes of ESRD remained unbalanced between the NHD and HD/HDF cohorts after matching, a post hoc sensitivity analysis with adjustments for cause of ESRD yielded similar results, supporting the conclusions of this study. Second, not all NHD patients could be matched to HD/HDF counterparts, and this study had to compromise somewhat on sample size and was underpowered to evaluate mortality. On the other hand, we gathered a large cohort of NHD patients that can be considered demographically representative for the Dutch NHD population. Third, as data on the $\mathrm{HD} / \mathrm{HDF}$ cohort were collected in a randomised trial as opposed to the observational NHD cohort, a potential risk of information bias exists, although minor as clinical parameters in the NHD 
cohort were assessed at standardised intervals as part of routine care. Also, we aimed to minimise interference due to different healthcare systems, by including patients on HD/HDF only when they were treated in Dutch dialysis centres. Fourth, the NHD cohort was drawn from only two centres; however, we deem the potential influence of individual prescribing practices (eg, nephrologists preferentially prescribing a single type of phosphate binder) insignificant, considering the equal number of phosphate-binding agents at baseline and the coinciding halving in number of pills in the NHD cohort, and otherwise uniform treatment patterns in the Netherlands. Fifth, the preferable method for hypertension monitoring is ambulatory blood pressure monitoring or self-reported home measurements, while we only measured predialysis blood pressure. Finally, our NHD cohort comprised patients who performed any kind of extended-hours NHD, which resulted in heterogeneity of frequency and location. On the other hand, we accounted for potential benefits of HDF treatment by comparing NHD with HD and HDF separately, that is, sensitivity analyses. Earlier data from CONTRAST showed that HDF helped to improve phosphate control, ${ }^{3738}$ yet not ESA resistance. ${ }^{39}$ These sensitivity analyses yielded similar results.

Overall, our study highlights the long-term improvements in important clinical parameters in patients switching to NHD, compared with conventional HD/HDF treatment. Translated to the clinic, our findings suggest that patients considering NHD can expect an increase in albumin, which is associated with lower mortality, ${ }^{40} \mathrm{a}$ lower dose of ESA and a sizeable reduction in pill burden, which is significant for their well-being. Our findings imply that NHD is more than a hype: it offers the prospect of enduring improvements for patients on HD, also suitable for patients expected to have a long waiting time for transplantation or those convicted to indefinite dialysis.

\author{
Author affiliations \\ ${ }^{1}$ Department of Nephrology and Hypertension, University Medical Centre Utrecht, \\ Utrecht, The Netherlands \\ ${ }^{2}$ Dialysis Centre Groningen, Groningen, The Netherlands \\ ${ }^{3}$ Division of Nephrology, Department of Internal Medicine, University Medical Centre, \\ Groningen, The Netherlands \\ ${ }^{4}$ Department of Nephrology, VU University Medical Centre, Amsterdam, The \\ Netherlands \\ ${ }^{5}$ Amsterdam Cardiovascular Sciences, VU University Medical Centre, Amsterdam, \\ The Netherlands \\ ${ }^{6}$ Julius Centre for Health Sciences and Primary Care, University Medical Centre \\ Utrecht, Utrecht, The Netherlands \\ ${ }^{7}$ Diapriva Dialysis Centre, Amsterdam, The Netherlands
}

Acknowledgements We thank Rolf $\mathrm{H} \mathrm{H}$ Groenwold for providing support in the statistical analyses.

Contributors TTJ, MPCG, TH, PJB, MLB and BCvJ contributed to the conception, design, analysis and interpretation of data. AÖ and MR collected part of the data. TTJ and BCvJ drafted the paper. All authors provided intellectual content of critical importance to the paper, and approved the final version of this paper and this submission.

Funding This research received no specific grant from any funding agency in the public, commercial or not-for-profit sectors.
Competing interests MPCG reports personal fees and non-financial support from Baxter Healthcare and personal fees from Fresenius Medical Care Netherlands, outside the submitted work.

Patient consent Detail has been removed from this case description/these case descriptions to ensure anonymity. The editors and reviewers have seen the detailed information available and are satisfied that the information backs up the case the authors are making.

Provenance and peer review Not commissioned; externally peer reviewed.

Data sharing statement No additional data are available.

Open Access This is an Open Access article distributed in accordance with the Creative Commons Attribution Non Commercial (CC BY-NC 4.0) license, which permits others to distribute, remix, adapt, build upon this work non-commercially, and license their derivative works on different terms, provided the original work is properly cited and the use is non-commercial. See: http://creativecommons.org/ licenses/by-nc/4.0/

(c) Article author(s) (or their employer(s) unless otherwise stated in the text of the article) 2018. All rights reserved. No commercial use is permitted unless otherwise expressly granted.

\section{REFERENCES}

1. Saran R, Li Y, Robinson B, et al. US Renal Data System 2015 Annual Data Report: Epidemiology of Kidney Disease in the United States. Am J Kidney Dis 2016;67:A7-8.

2. Pippias M, Jager KJ, Kramer A, et al. The changing trends and outcomes in renal replacement therapy: data from the ERA-EDTA Registry. Nephrol Dial Transplant 2016;31:831-41.

3. Go AS, Chertow GM, Fan D, et al. Chronic kidney disease and the risks of death, cardiovascular events, and hospitalization. $N$ Engl J Med 2004;351:1296-305.

4. Burton JO, Jefferies HJ, Selby NM, et al. Hemodialysis-induced repetitive myocardial injury results in global and segmental reduction in systolic cardiac function. Clin J Am Soc Nephrol 2009;4:1925-31.

5. Flythe JE, Kimmel SE, Brunelli SM. Rapid fluid removal during dialysis is associated with cardiovascular morbidity and mortality. Kidney Int 2011;79:250-7.

6. Sands JJ, Usvyat LA, Sullivan T, et al. Intradialytic hypotension: frequency, sources of variation and correlation with clinical outcome. Hemodial Int 2014;18:415-22.

7. Palmer SC, Hayen A, Macaskill P, et al. Serum levels of phosphorus, parathyroid hormone, and calcium and risks of death and cardiovascular disease in individuals with chronic kidney disease: a systematic review and meta-analysis. JAMA 2011;305:1119-27.

8. Shanahan CM, Crouthamel MH, Kapustin A, et al. Arterial calcification in chronic kidney disease: key roles for calcium and phosphate. Circ Res 2011;109:697-711.

9. London GM, Guérin AP, Marchais SJ, et al. Arterial media calcification in end-stage renal disease: impact on all-cause and cardiovascular mortality. Nephrol Dial Transplant 2003;18:1731-40.

10. Ketteler M, Biggar PH. Review article: Getting the balance right: assessing causes and extent of vascular calcification in chronic kidney disease. Nephrology 2009;14:389-94.

11. Chazot C, Ok E, Lacson E, et al. Thrice-weekly nocturnal hemodialysis: the overlooked alternative to improve patient outcomes. Nephrol Dial Transplant 2013;28:2447-55.

12. Cornelis T, van der Sande FM, Eloot S, et al. Acute hemodynamic response and uremic toxin removal in conventional and extended hemodialysis and hemodiafiltration: a randomized crossover study. Am J Kidney Dis 2014;64:247-56.

13. Culleton BF, Walsh M, Klarenbach SW, et al. Effect of frequent nocturnal hemodialysis vs conventional hemodialysis on left ventricular mass and quality of life: a randomized controlled trial. JAMA 2007;298:1291-9.

14. Rocco MV, Lockridge RS, Beck GJ, et al. The effects of frequent nocturnal home hemodialysis: the Frequent Hemodialysis Network Nocturnal Trial. Kidney Int 2011;80:1080-91.

15. Chan CT, Floras JS, Miller JA, et al. Regression of left ventricular hypertrophy after conversion to nocturnal hemodialysis. Kidney Int 2002;61:2235-9.

16. David S, Kümpers P, Eisenbach GM, et al. Prospective evaluation of an in-centre conversion from conventional haemodialysis to an intensified nocturnal strategy. Nephrol Dial Transplant 2009;24:2232-40. 
17. Lacson $\mathrm{E}$, Wang W, Lester $\mathrm{K}$, et al. Outcomes associated with incenter nocturnal hemodialysis from a large multicenter program. Clin J Am Soc Nephrol 2010;5:220-6.

18. Bugeja A, Dacouris N, Thomas A, et al. In-center nocturnal hemodialysis: another option in the management of chronic kidney disease. Clin J Am Soc Nephrol 2009;4:778-83.

19. Ok E, Duman S, Asci G, et al. Comparison of 4- and 8-h dialysis sessions in thrice-weekly in-centre haemodialysis: a prospective, case-controlled study. Nephrol Dial Transplant 2011;26:1287-96.

20. Lacson E, Xu J, Suri RS, et al. Survival with three-times weekly in-center nocturnal versus conventional hemodialysis. J Am Soc Nephrol 2012;23:687-95.

21. Rocco MV, Daugirdas JT, Greene T, et al. Long-term Effects of Frequent Nocturnal Hemodialysis on Mortality: The Frequent Hemodialysis Network (FHN) Nocturnal Trial. Am J Kidney Dis 2015;66:459-68.

22. Penne EL, Blankestijn PJ, Bots ML, et al. Effect of increased convective clearance by on-line hemodiafiltration on all cause and cardiovascular mortality in chronic hemodialysis patients - the Dutch CONvective TRAnsport STudy (CONTRAST): rationale and design of a randomised controlled trial [ISRCTN38365125]. Curr Control Trials Cardiovasc Med 2005;6:8.

23. Clase CM, St Pierre MW, Churchill DN. Conversion between bromcresol green- and bromcresol purple-measured albumin in renal disease. Nephrol Dial Transplant 2001;16:1925-9.

24. World Health Organization. ATC/DDD Index. 2016 http://www.whocc. no/atc_ddd_index/ (accessed 5 Jun 2016).

25. Grooteman MP, van den Dorpel MA, Bots ML, et al. Effect of online hemodiafiltration on all-cause mortality and cardiovascular outcomes. J Am Soc Nephrol 2012;23:1087-96.

26. Bates D, Mächler M, Bolker B, et al. Fitting Linear Mixed-Effects Models Using Ime4. J Stat Softw 2015;67:1-48.

27. Therneau TM, Grambsch PM. Modeling survival data: extending the cox model. New York: Springer, 2000.

28. Mitra R, Reiter JP. A comparison of two methods of estimating propensity scores after multiple imputation. Stat Methods Med Res 2016;25:188-204.
29. Buuren S, Groothuis-Oudshoorn K. mice : Multivariate Imputation by Chained Equations in R. J Stat Softw 2011:45:1-67.

30. De H, Imai K, King G, et al. Matchlt: Nonparametric Preprocessing for Parametric Causal Inference. J Stat Softw 2011:42:1-28

31. Rubin DB, Schenker N. Multiple Imputation for Interval Estimation from Simple Random Samples with Ignorable Nonresponse. J Am Stat Assoc 1986;81:366-74.

32. R Core Team. R: a language and environment for statistical computing. Vienna, Austria: R Foundation for Statistical Computing, 2016.

33. Leypoldt JK, Jaber BL, Zimmerman DL. Predicting treatment dose for novel therapies using urea standard Kt/V. Semin Dial 2004:17:142-5.

34. Jardine MJ, Zuo L, Gray NA, et al. A Trial of Extending Hemodialysis Hours and Quality of Life. J Am Soc Nephrol 2017;28:1898-911.

35. Kidney Disease: Improving Global Outcomes (KDIGO) CKDMBD Work Group. KDIGO clinical practice guideline for the diagnosis, evaluation, prevention, and treatment of Chronic Kidney Disease-Mineral and Bone Disorder (CKD-MBD). Kidney Int Suppl 2009:S1-30.

36. Rivara MB, Adams SV, Kuttykrishnan S, et al. Extended-hours hemodialysis is associated with lower mortality risk in patients with end-stage renal disease. Kidney Int 2016;90:1312-20.

37. Penne EL, van der Weerd NC, van den Dorpel MA, et al. Short-term effects of online hemodiafiltration on phosphate control: a result from the randomized controlled Convective Transport Study (CONTRAST). Am J Kidney Dis 2010;55:77-87.

38. den Hoedt $\mathrm{CH}$, Bots ML, Grooteman MP, et al. Online hemodiafiltration reduces systemic inflammation compared to lowflux hemodialysis. Kidney Int 2014;86:423-32.

39. van der Weerd NC, Den Hoedt CH, Blankestijn PJ, et al. Resistance to erythropoiesis stimulating agents in patients treated with online hemodiafiltration and ultrapure low-flux hemodialysis: results from a randomized controlled trial (CONTRAST). PLoS One 2014:9:e94434.

40. de Roij van Zuijdewijn CL, ter Wee PM, Chapdelaine I, et al. A Comparison of 8 Nutrition-Related Tests to Predict Mortality in Hemodialysis Patients. J Ren Nutr 2015;25:412-9. 